\title{
Identification of three MAPKKKs forming a linear signaling pathway leading to programmed cell death in Nicotiana benthamiana
}

Masayoshi Hashimoto, Ken Komatsu, Kensaku Maejima, Yukari Okano, Takuya Shiraishi, Kazuya Ishikawa, Yusuke Takinami, Yasuyuki Yamaji and Shigetou Namba*

\begin{abstract}
Background: The mitogen-activated protein kinase (MAPK) cascade is an evolutionarily ancient mechanism of signal transduction found in eukaryotic cells. In plants, MAPK cascades are associated with responses to various abiotic and biotic stresses such as plant pathogens. MAPK cascades function through sequential phosphorylation: MAPK kinase kinases (MAPKKKs) phosphorylate MAPK kinases (MAPKKs), and phosphorylated MAPKKs phosphorylate MAPKs. Of these three types of kinase, the MAPKKKs exhibit the most divergence in the plant genome. Their great diversity is assumed to allow MAPKKKs to regulate many specific signaling pathways in plants despite the relatively limited number of MAPKKs and MAPKs. Although some plant MAPKKKs, including the MAPKKKa of Nicotiana benthamiana (NbMAPKKKa), are known to play crucial roles in plant defense responses, the functional relationship among MAPKKK genes is poorly understood. Here, we performed a comparative functional analysis of MAPKKKs to investigate the signaling pathway leading to the defense response.
\end{abstract}

Results: We cloned three novel MAPKKK genes from N. benthamiana: NbMAPKKKKB, NbMAPKKKY, and NbMAPKKKG 2. Transient overexpression of full-length NbMAPKKK $\beta$ or NbMAPKKKY or their kinase domains in N. benthamiana leaves induced hypersensitive response (HR)-like cell death associated with hydrogen peroxide production. This activity was dependent on the kinase activity of the overexpressed MAPKKK. In addition, virus-induced silencing of NbMAPKKK $\beta$ or NbMAPKKKY expression significantly suppressed the induction of programmed cell death (PCD) by viral infection. Furthermore, in epistasis analysis of the functional relationships among NbMAPKKK $\beta$, NbMAPKKKY, and NbMAPKKKa (previously shown to be involved in plant defense responses) conducted by combining transient overexpression analysis and virus-induced gene silencing, silencing of NbMAPKKKa suppressed cell death induced by the overexpression of the NbMAPKKKK kinase domain or of NbMAPKKKY, but silencing of NbMAPKKK $\beta$ failed to suppress cell death induced by the overexpression of NbMAPKKKa or NbMAPKKKY. Silencing of NbMAPKKKY suppressed cell death induced by the NbMAPKKK $\beta$ kinase domain but not that induced by NbMAPKKKa.

Conclusions: These results demonstrate that in addition to NbMAPKKKa, NbMAPKKK $\beta$ and NbMAPKKKY also function as positive regulators of PCD. Furthermore, these three MAPKKKs form a linear signaling pathway leading to PCD; this pathway proceeds from NbMAPKKK $\beta$ to NbMAPKKKy to NbMAPKKKa.

\footnotetext{
* Correspondence: anamba@mail.ecc.u-tokyo.ac.jp

Department of Agricultural and Environmental Biology, Graduate School of Agricultural and Life Sciences, The University of Tokyo, 1-1-1 Yayoi, Bunkyoku, Tokyo 113-8657, Japan
} 


\section{Background}

Because plants lack an adaptive immune system, appropriate perceptions and responses of individual cells to various environmental stimuli, such as the biotic stress caused by phytopathogenic microorganisms, are critically important. The plant defense response against biotic stress is triggered by the recognition of conserved pathogen-associated molecular patterns (PAMPs) or of pathogen strain-specific factors known as elicitors or effectors [1]. The response triggered by PAMPs is known as the basal defense response, whereas that triggered by specific elicitors is known as the hypersensitive response (HR). In the latter, an effector is recognized by a corresponding plant resistance ( $R$ ) protein. The HR is frequently accompanied by programmed cell death (PCD), which plays a particularly important role in the defense against biotrophic pathogens but is also an essential function in normal plant development and differentiation [2]. Although many plant components required for the PCD-associated HR have been identified, the entire signaling pathway leading to PCD has not been elucidated.

The mitogen-activated protein kinase (MAPK) cascade is a highly evolutionarily conserved signal transduction mechanism found in eukaryotic cells. Subsequent to activation of the cascade by various extracellular stimuli, the signal is transduced intracellularly by sequential phosphorylation. In plants, MAPK cascades are associated with developmental and hormonal responses and with stress responses to abiotic and biotic factors [3]. A MAPK cascade consists of three functionally linked protein kinases: a MAPK is phosphorylated and activated by a MAPK kinase (MAPKK), which is in turn activated by an upstream MAPK kinase kinase (MAPKKK). Typical MAPK substrates are cytoplasmic or nuclear proteins, such as transcription factors [3]. MAPKKKs are the most divergent of these three types of kinases in plants; the Arabidopsis thaliana genome contains approximately 60 MAPKKKs, 10 MAPKKs, and 20 MAPKs [4].

Based on phylogenetic analysis of the amino acid sequences of their catalytic kinase domains, plant MAPKKKs have been classified into three groups: A, B, and $C$ [4]. Group A contains many MAPKKKs involved in PCD and stress and defense responses; e.g., A. thaliana AtMEKK1 is involved in the signaling pathway of basal defense induced by PAMPs [5], and Medicago sativa MsOMTK1 [6] is involved in that of oxidative stressinduced cell death. Group A also includes MAPKKKs that have important functions in HR induction. Silencing of the genes encoding Nicotiana tabacum NPK1 (NtNPK1) and

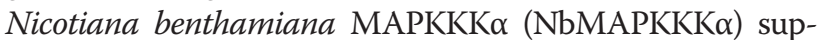
presses the $N$ gene-mediated HR induced by the helicase domain of tobacco mosaic virus (TMV) replicase and Ptomediated HR induced by Pseudomonas syringae pv. tomato (Pst) effector avrPto, respectively [7,8]. Recently, $N$. benthamiana NbMAPKKKe and its tomato (Solanum lycopersicum) ortholog SIMAPKKKe have been implicated in PCD induction in the HR against Gram-negative bacterial pathogens [9]. In addition, silencing of the genes encoding the MAPKK MEK2 and the MAPK SIPK, both of which act downstream of NbMAPKKK $\alpha$, also attenuates the $N$ gene-mediated HR against TMV [10]. Conversely, silencing of the tomato orthologs of MAPKK MEK1 and MAPK NTF6, both of whose tobacco orthologs act downstream of NtNPK1in tobacco, leads to loss of the Pto-mediated HR in tomato [11]. Therefore, the NtNPK1- and NbMAPKKK $\alpha-$ initiated MAPK cascades are essential for both the $N$ genemediated and the Pto-mediated HR, suggesting that at least two distinct MAPK cascades are involved in the regulation of a single HR event [8]. Furthermore, it is now becoming apparent that two distinct MAPK cascades are involved in non-HR environmental responses [12].

Plants generally appear to use the same MAPKK/ MAPK sets in different responses to environmental stimuli. The $A$. thaliana MAPKKs AtMKK4 and AtMKK5 and/or their downstream component MPK6 are involved not only in the signaling pathway for basal defense downstream of AtMEKK1 but also in ethylene production and stomata formation [5,13,14]. Given the relatively limited number of MAPKKs and MAPKs in plants, the diversity of these responses (functions) is assumed to be possible due to the great diversity of MAPKKKs $[15,16]$. Therefore, comparative functional analysis among MAPKKKs is needed to reveal the molecular mechanisms underlying a variety of responses to environmental stresses.

We previously showed that systemic necrosis, the disease symptom caused by plantago asiatica mosaic virus Li1 (PlAMV-Li1), was accompanied by resistance traits similar to HR. Using tobacco rattle virus (TRV)-based virus-induced gene silencing (VIGS) [17], we demonstrated that NbSGT1 and NbRAR1, which are important in the HR, and the MAPK cascade including NbMAPKKK $\alpha /$ $\mathrm{NbMEK} 2$, are essential for the induction of PCD-associated systemic necrosis induced by PlAMV-Li1 $[18,19]$. This result and those described above led us to hypothesize that other MAPKKK genes in addition to NbMAPKKKa are involved in the systemic necrosis induced by PlAMV-Li1.

In the present study, we isolated three novel group A MAPKKK genes from $N$. benthamiana, a model plant of the family Solanaceae, using an expressed sequence-tag (EST) database. The three cloned genes were designated NbMAPKKK $\beta, N b M A P K K K \gamma$, and NbMAPKKK€2. Further study revealed that NbMAPKKK $\beta$ and NbMAPKKK $\gamma$ are positive regulators of PCD. In addition, the results of epistasis analysis performed using VIGS and agroinfiltration suggest that two of these MAPKKKs (NbMAPKKK $\beta$ and NbMAPKKK $\gamma$ ), together with NbMAPKKK $\alpha$, comprise a linear signaling pathway important in the induction of PCD. 


\section{Results}

\section{Cloning of three novel group A MAPKKK genes from Nicotiana benthamiana}

To conduct a comparative analysis of the roles of MAPKKK genes in defense responses, we first sought to clone $N$. benthamiana MAPKKK genes belonging to group A. Group A includes four subgroups, A1-A4, and contains many genes involved in plant defense responses. To obtain one $N$. benthamiana MAPKKK gene homolog from each group A subgroup, we selected the following: the Arabidopsis MAPKKK genes AtMEKK1 [20], AtMAPKKK $\gamma$, and AtMAPKKK $\in 1[21,22]$ as representatives of the A1, A2, and A4 subgroups, respectively. For the A3 subgroup, which includes NtNPK1 [23] and AtANP1, we were able to amplify $N$. benthamiana cDNA fragments using NtNPK1-specific primers; the amplified gene turned out to be $98.0 \%$ identical in nucleotide sequence to that encoding the NtNPK1 kinase domain, which has been well characterized for its role in defense responses [7]. Therefore, the A3 subgroup was excluded from further study.

Using the nucleotide sequences encoding the highly conserved kinase domains of AtMEKK1 [19], AtMAPKKK $\gamma$, and AtMAPKKK $€ 1$ as queries for BLAST searches against the $N$. benthamiana, $N$. tabacum, and Solanum lycopersicum EST databases, we obtained three EST sequences: $N$. benthamiana TC15397, N. tabacum BP133312, and S. lycopersicum BI931567, respectively. Based on these sequences, we designed specific primers to isolate full-length $N$. benthamiana MAPKKK cDNA clones, as described in the Materials and Methods. The cloned $N$. benthamiana MAPKKK genes are predicted to encode proteins with highly conserved kinase domains and more divergence in other regions, a general feature of plant MAPKKK genes [4].

Next, we used the amino acid sequences of the kinase domains of our newly cloned MAPKKKs and those of other previously identified MAPKKKs to construct a phylogenetic tree (Figure 1A). Our newly cloned MAPKKK genes were tentatively designated after the name of the most closely related genes in the phylogenetic tree. Thus, our A2 MAPKKK homolog was designated $N b M A P K K K \gamma$ after A. thaliana AtMAPKKK $\gamma$. At a late phase of this study, an A4 MAPKKK homolog, N. benthamiana NbMAPKKKє, was reported [9]. Although our A4 homolog shares high sequence similarity with $N b M A P K K K \epsilon$, the genes were not identical $(96.6 \%$ or $95.0 \%$ identity at the nucleotide or amino acid level, respectively) and differed in length. Because these results indicated that A4 MAPKKK homologs in $N$. benthamiana consisted of at least two genes, our A4 homolog was designated $N b M A P K K K \epsilon 2$ after $N b M A P K K K \epsilon$. In fact, from the recently released $N$. benthamiana draft genome sequence (http://solgenomics. net/), only two contigs that show high sequence homology (about $~ 90 \%$ ) with $N b M A P K K K \epsilon 2$ were retrieved and each of these sequences corresponded to $N b M A P K K K \epsilon 2$ and
NbMAPKKKє, respectively. Although our A1 MAPKKK homolog was most closely related to MsOMTK1, which was designated after the functional features of its gene products, designating this gene "NbOMTK1" was apparently incorrect, as we do not know if our A1 homologous gene product and MsOMTK1 have a similar function. The next most closely related genes were AtMEKK1 and Brassica napus BnMAPKKK $\beta 1$. Therefore, our A1 homolog was designated $N b M A P K K K \beta$ after $B n M A P K K K \beta 1$, a gene of the A1 subgroup, in accordance with other newly cloned genes $N b M A P K K K \gamma$ and $N b M A P K K K \epsilon 2$. The NbMAPKKK $\beta$, $N b M A P K K K \gamma$, and NbMAPKKK€2 cDNA sequences determined in this study were deposited in the DNA Data Bank of Japan (DDBJ) under the accession numbers AB649283, AB649284, and AB649285, respectively.

The domain structures of the three novel MAPKKK genes are shown in Figure 1B. The $N b M A P K K K \beta$, $N b M A P K K K \gamma$, and NbMAPKKKє2 cDNA are 2477, 2794, and $4708 \mathrm{bp}$ in length, respectively, and are respectively predicted to encode proteins of 564, 651, and 1395 amino acids. Kinase domains are found in the Cterminal regions of $\mathrm{NbMAPKKK} \beta$ and NbMAPKKK $\gamma$ and the N-terminal region of NbMAPKKKє2. The Cterminal region of NbMAPKKK $€ 2$ contains two ARM (armadillo/ $\beta$-catenin-like repeat) domains. The domain structures of these proteins are similar to those of their respective $A$. thaliana homologs.

\section{Overexpression of the NbMAPKKK $\beta$ and NbMAPKKK $\gamma$ kinase domains, but not the NbMAPKKKع2 kinase domain, causes cell death}

Overexpression of the kinase domain of the tomato homolog of NbMAPKKK $\alpha$, SIMAPKKK $\alpha$, in $N$. benthamiana leaves by agroinfiltration has been reported to induce pathogen-independent cell death [8]. To investigate whether our three newly identified MAPKKKs also possessed cell death-inducing activity, we performed transient overexpression analysis of each kinase domain using agroinfiltration. The transient overexpression of the kinase domains of NbMAPKKK $\beta$ and NbMAPKKK $\gamma$ induced pathogen-independent cell death in the infiltrated area (Figure 2A). In the infiltrated areas, cell death was associated with significant hydrogen peroxide production, which is detected by a characteristic brown color that emerges upon 3,3'-diaminobenzidine (DAB) staining. These results were confirmed by measuring the extent of cell death using an ion leakage assay (Figure 2B), which showed significantly increased ion leakage in the NbMAPKKK $\beta$ - and NbMAPKKK $\gamma$-overexpressing areas. No significant difference in the level of ion leakage was observed for NbMAPKKK $\beta$ vs. NbMAPKKK $\gamma$. In contrast, overexpression of the kinase domain of NbMAPKKK 22 failed to induce cell death or hydrogen peroxide production and failed to increase the level of ion leakage. 
A

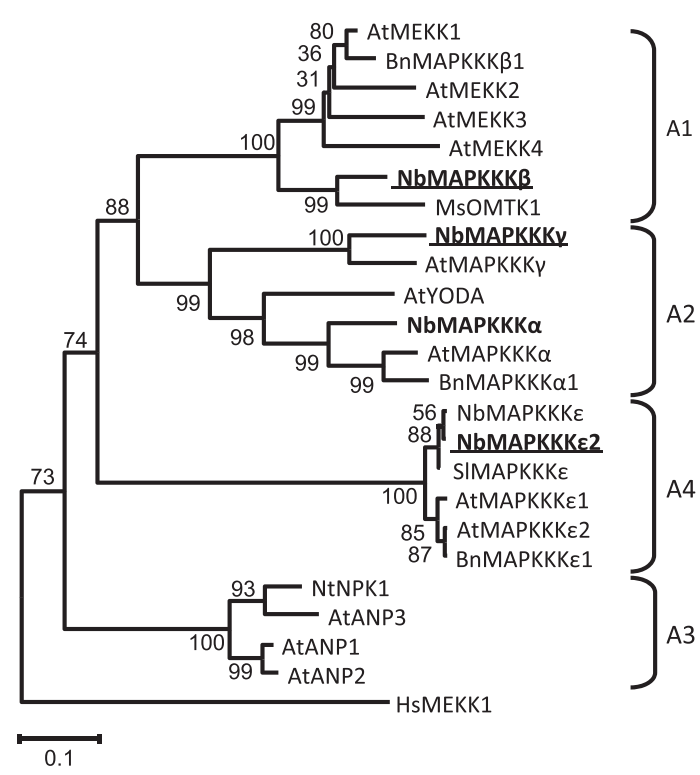

B

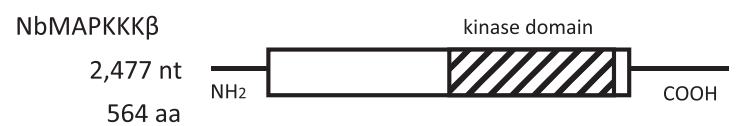

NbMAPKKKY

2,794 nt

651 aa

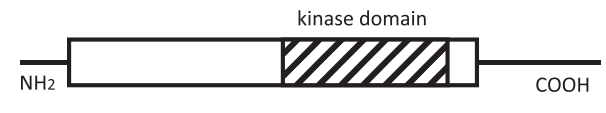

NbMAPKKKE2

$4,708 \mathrm{nt}$

1,394 aa

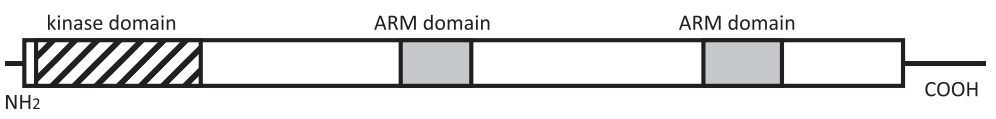

Figure 1 Phylogenetic analysis of plant MAPKKK genes and structural features of novel N. benthamiana MAPKKK genes. A) Phylogenetic tree for the kinase domain-encoding regions of group A MAPKKK genes. Numbers represent bootstrap scores. Names of genes used in this study are shown in boldface. Names of the three novel MAPKKK genes are underlined. B) Diagram of the domain structures of the three novel MAPKKK genes. ORFs are indicated by boxes. The 5'- and 3'-untranslated regions (UTRs) are shown as horizontal lines at the left and right, respectively, of the boxes. Shaded boxes and gray boxes indicate kinase domains and ARM domains, respectively. ORFs and UTRs are drawn to the same scale.

Western blot analysis using anti-Myc monoclonal antibody (Millipore, Billerica, MA, USA) was performed to assess the expression levels of these kinase domains (Figure 2C). Specific signals of NbMAPKKK $\beta$ and NbMAPKKK€2 kinase domains were detected, but no accumulation of NbMAPKKK $\gamma$ kinase domain was found. However, we suppose that the NbMAPKKK $\gamma$ kinase domain was accurately expressed, even at very low levels, because its overexpression rapidly induced cell death. Western blot analysis also showed that the accumulated expression level of NbMAPKKK€2 kinase domain, whose overexpression did not induce cell death, was much greater than the expression levels of the NbMAPKKK $\beta$ and NbMAPKKK $\gamma$ kinase domains. Because these results indicated that NbMAPKKKE2 is not involved in the induction of pathogen-independent cell death, we excluded NbMAPKKKE2 from further analysis.

\section{Overexpression of full-length, catalytically active} NbMAPKKK $\beta$ or NbMAPKKK $\gamma$ causes cell death

To further investigate the involvement of NbMAPKKK $\beta$ and NbMAPKKK $\gamma$ in pathogen-independent cell death, we conducted transient overexpression experiments using their full-length open reading frames (ORFs). As shown in Figure 2D, overexpression of NbMAPKKK $\beta$ or NbMAPKKK $\gamma$ induced cell death. The observed level of ion leakage was similar for overexpression of $\mathrm{NbMAPKKK} \beta$, NbMAPKKK $\gamma$, and NbMAPKKK $\alpha$, which was used as a positive control (Figure 2B).

Several previous studies have demonstrated that kinase activity is required for the triggering of pathogenindependent cell death by transient overexpression of a component of a MAPK cascade $[8,24,25]$. To examine whether NbMAPKKK $\beta$ - and NbMAPKKK $\gamma$-induced cell death also required kinase activity, we constructed full- 

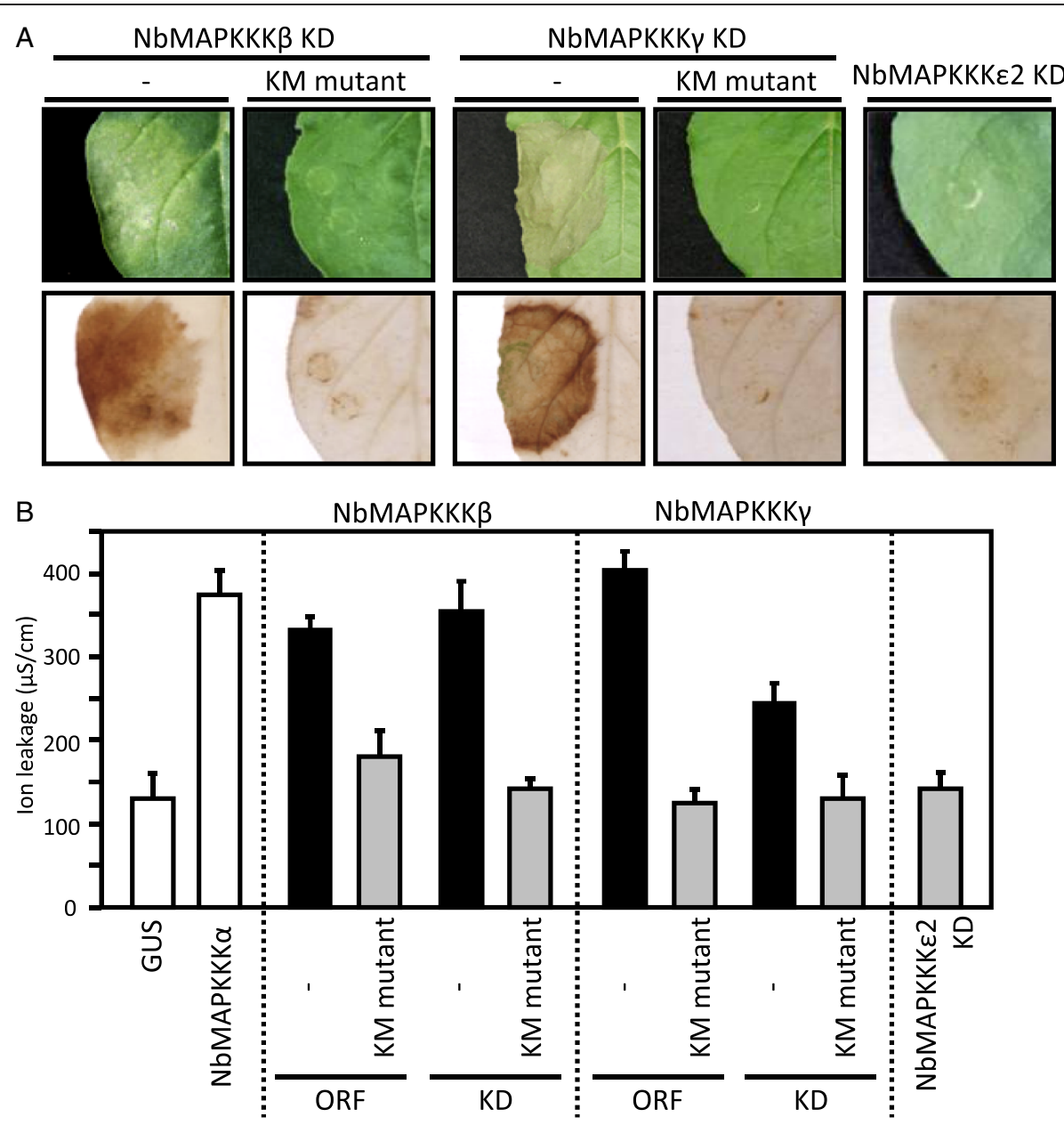

C

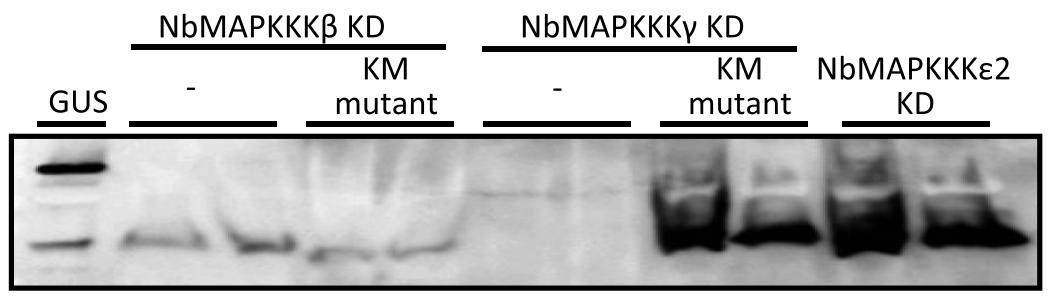

D

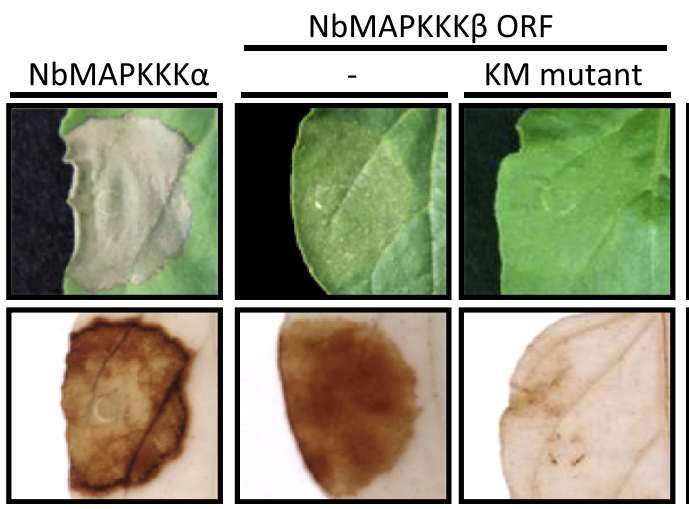

NbMAPKKKY ORF

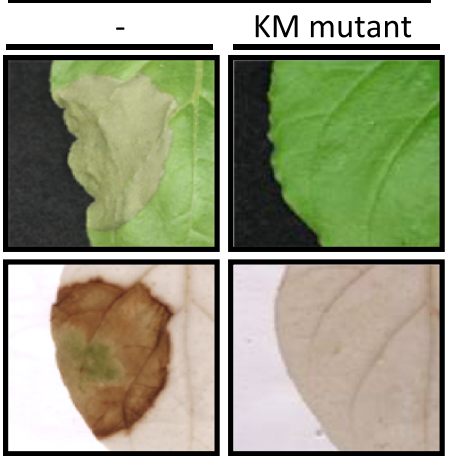

Figure 2 (See legend on next page.) 


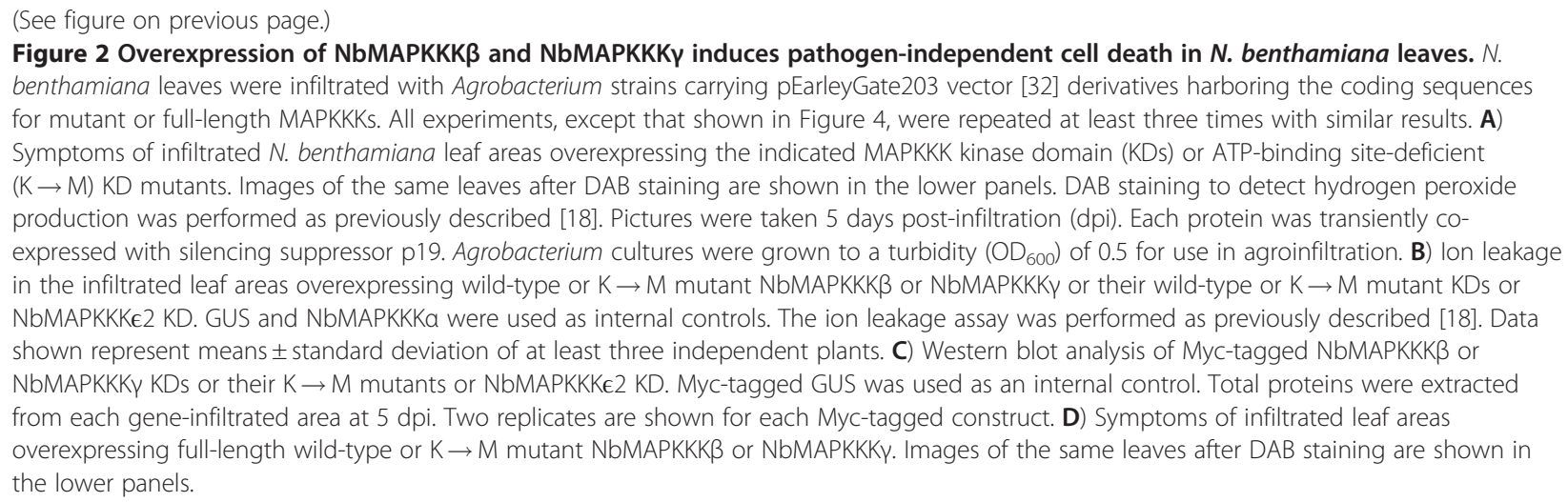

length and kinase domain MAPKKK mutants deficient in ATP binding. In these mutants, the essential conserved lysine residue (K) in the ATP-binding site of the kinase domain was replaced with a methionine $(M)$ [8]. As shown in Figure $2 \mathrm{~A}$ and $2 \mathrm{D}$, none of these $\mathrm{K} \rightarrow \mathrm{M}$ mutants induced cell death when overexpressed. This result was confirmed by DAB staining (Figure $2 \mathrm{~A}$ and $2 \mathrm{D}$ ) and ion leakage assays (Figure 2B). Also, the expression of these kinase domain $\mathrm{K}$ $\rightarrow \mathrm{M}$ mutants was confirmed by western blot analysis with anti-Myc monoclonal antibody (Figure 2C). These results indicate that cell death induced by the transient overexpression of $N$. benthamiana NbMAPKKK $\beta$ or NbMAPKKK $\gamma$ is dependent on their kinase activities.

\section{Silencing of NbMAPKKK $\beta$ or NbMAPKKKY $\gamma$ suppresses PIAMV-Li1-induced PCD}

In light of the above finding that overexpression of fulllength NbMAPKKK $\beta$ or NbMAPKKK $\gamma$ or their kinase domains can induce cell death, we expected that silencing of the genes encoding these MAPKKKs would suppress virally induced PCD. Therefore, we used a TRVbased VIGS system [17] to silence NbMAPKKK $\beta$ and NbMAPKKK $\gamma$. Successful silencing of each gene was confirmed by analyzing the expression of $N b M A P K K K \beta$, NbMAPKKK $\gamma$, and NbMAPKKKa (control) using realtime reverse transcription (RT)-PCR analysis (Figure 3A). Also, Southern blot analyses using a kinase domainspecific probe of each gene revealed that $N b M A P K K K \beta$ and $N b M A P K K K \gamma$ exist as single-copy and multiple-copy genes, respectively, in the $N$. benthamiana genome (Additional file 1: Figure S1). In the $N$. benthamiana draft genome, two contigs showing high sequence homology (about $>90 \%$ ) with $N b M A P K K K \gamma$ were obtained, suggesting that there are at least two copies of $N b M A P K K K \gamma$ homologs. Thus, we assume that a single gene is specifically targeted in $N b M A P K K K \beta$-silenced plants, but one or more highly similar genes might be targeted in NbMAPKKK $\gamma$ silenced plants. Hereafter, we use the term "NbMAPKKKY" to indicate $N b M A P K K K \gamma$ and/or its homologous gene(s) except for transient overexpression experiments. The genesilenced plants did not display any obvious phenotypic differences compared with non-silenced (VIGS vector) control plants (Figure 3B), suggesting that neither NbMAPKKK $\beta$ nor NbMAPKKKY is involved in normal plant growth and development. In contrast, silencing of NtNPK1, a MAPKKK involved in cell plate formation in plant cytokinesis and in $N$ gene-mediated HR cell death, causes severe stunting of plants [7].

$N b M A P K K K \beta$ - and NbMAPKKK $\gamma$-silenced plants and control non-silenced plants were subsequently inoculated with PIAMV-Li1, which induces PCD-associated necrosis in $N$. benthamiana. In the PIAMV-Li1-infiltrated areas of non-silenced plants, we observed characteristic necrotic symptoms and brown color staining, indicating the accumulation of hydrogen peroxide. However, in the Li1infiltrated $N b M A P K K K \beta$-silenced plants, cell death and hydrogen peroxide production were completely compromised. Also, in $N b M A P K K K \gamma$-silenced plants, cell death and hydrogen peroxide production were suppressed completely and partially, respectively, despite only about $20 \%$ decrease in the abundance of $N b M A P K K K \gamma$ transcripts (Figure 3C). Suppression of cell death in the NbMAPKKK $\beta$ and $N b M A P K K K \gamma$-silenced plants was confirmed using ion leakage assays (Figure 3D). The suppression of cell death in $N b M A P K K K \gamma$-silenced plants despite the slight decrease in its mRNA can be explained by a strict requirement of a high level of NbMAPKKK $\gamma$ protein in cell death. Although it is possible that residual NbMAPKKK $\gamma$ protein contributes to the decreased level of hydrogen peroxide production, its level might be too low to induce cell death. These results indicate that NbMAPKKK $\beta$ and NbMAPKKK $\gamma$ are involved in hydrogen peroxide production and $\mathrm{PCD}$ induced by PlAMV-Li1.

\section{NbMAPKKKa, NbMAPKKK $\beta$, and NbMAPKKK $\beta$ form a linear signaling pathway that induces cell death} Together with the findings of our previous study showing that NbMAPKKK $\alpha$ is involved in PlAMV-Li1- 


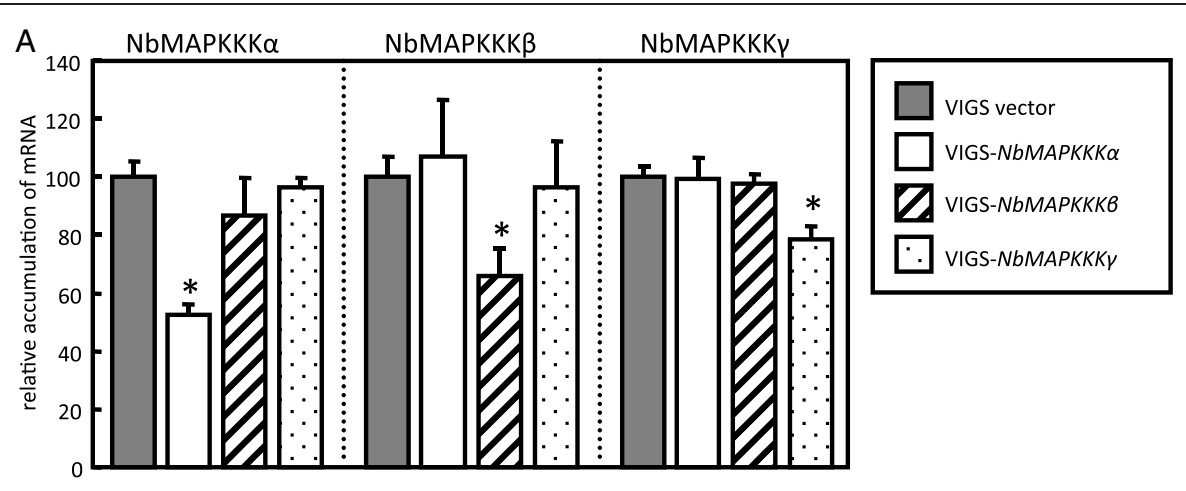

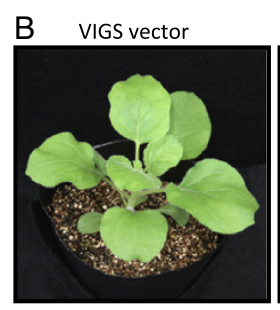

$\underline{\mathrm{C}}$

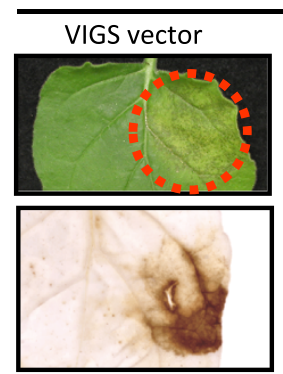

VIGS-NbMAPKKKY

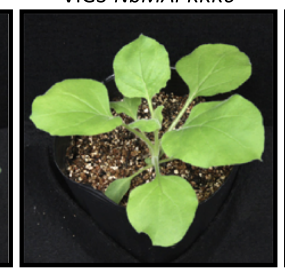

Li1
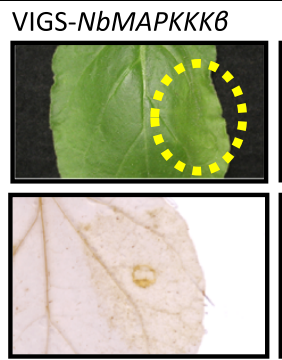

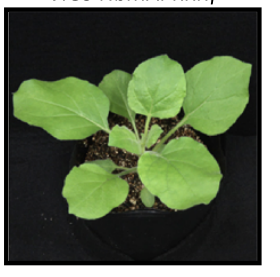

VIGS-NbMAPKKKY
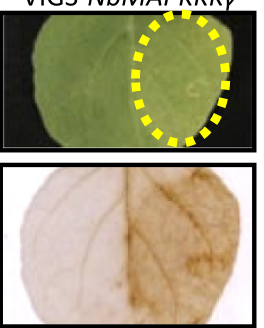

$\mathrm{D}$
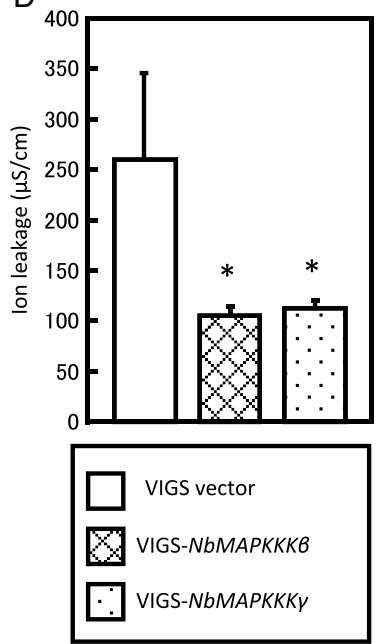

Figure 3 Silencing of NbMAPKKK $\beta$ and NbMAPKKKY suppresses PIAMV-Li1-induced PCD. A) Confirmation of specific MAPKKK gene silencing in gene-silenced plants. The relative quantities of NbMAPKKKa, NbMAPKKKB, and NbMAPKKKY mRNA in non-silenced or NbMAPKKKa-, NbMAPKKKB-, or NbMAPKKKY-silenced plants were measured using real-time RT-PCR. The data for each leaf tissue sample were normalized to the Nb18S rRNA gene expression level in the same sample. Data shown represent means \pm standard deviation of at least three independent plants. Asterisks indicate statistically significant differences from control plants $(P<0.05)$. B) Typical phenotypes observed in non-silenced and NbMAPKKKK- and NbMAPKKKY-silenced plants. Pictures were taken 21 dpi. C) Symptoms in the PIAMV-Li1-infiltrated areas of non-silenced and NbMAPKKKK- or NbMAPKKKY-silenced plants (upper panels) and DAB staining of the same leaves (lower panels). Red circles indicate cell death; yellow circles indicate no symptoms. Leaves were infiltrated with a PIAMV-Li1-expressing Agrobacterium culture grown to a turbidity $\left(\mathrm{OD}_{600}\right)$ of 0.05 . D) lon leakage in the PIAMV-Li1-infiltrated areas of non-silenced and NbMAPKKKB- or NbMAPKKKY- silenced plants. Data shown represent means \pm standard deviation of at least three independent plants. Asterisks indicate statistically significant differences from control plants $(P<0.05)$.

induced PCD [19], the above findings demonstrate that $N b M A P K K K \beta$ and $N b M A P K K K \gamma$ are also essential for this virally induced PCD. To determine the relationships among these three genes in the PCD signaling pathway, we designed epistasis experiments combining transient overexpression and silencing of combinations of these genes. In these experiments, plants with a specific VIGSsilenced MAPKKK gene were agroinfiltrated with an Agrobacterium culture expressing a different MAPKKK gene, and the level of ion leakage was measured. Prior to the epistasis experiments, a preliminary experiment was performed to determine the minimum turbidity of Agrobacterium inoculum for each gene sufficient to induce complete cell death. Complete cell death was observed at the following cell densities at $\mathrm{OD}_{600}: 0.5$, NbMAPKKKa; 1.0, NbMAPKKK $\beta$; and 0.05, NbMAPKKK $\gamma$. Although each MAPKKK was overexpressed at different concentrations of Agrobacterium inocula, no significant difference in the basal ion leakage level was observed among the different turbidities (ranging from 0.05 to 1.0) of Agrobacterium inocula that expressed the GUS gene in wild-type plants (Additional file 2: Figure S2). For NbMAPKKK $\beta$, cell death induced by both the full-length ORF and kinase domain was slow and weak, but cell death induced by the kinase domain was more often observed than that induced by the full-length ORF. In the ion leakage assay shown in Figure $2 \mathrm{~B}$, this tendency was detected, although the difference in cell death induced by the full-length ORF and 
kinase domain of NbMAPKKK $\beta$ was not statistically significant. Previous reports have shown that the substrate specificity of MAPKKK is not affected when only the kinase domain is transiently activated $[5,8,26]$. Hence, the kinase domain of NbMAPKKK $\beta$ was used for further analysis, instead of the full-length ORF.

As shown in Figure 4A, ion leakage levels induced by transient expression of the NbMAPKKK $\beta$ kinase domain or full-length NbMAPKKK $\gamma$ were lower in $N b M A P K K K \alpha$ silenced plants than in VIGS vector-only control plants. Cell death induced by both NbMAPKKK $\beta$ and NbMAPKKK $\gamma$ was consistently significantly suppressed in NbMAPKKK $\alpha$-silenced plants compared to control plants (data not shown). This result suggests that NbMAPKKK $\alpha$ either acts downstream of both NbMAPKKK $\beta$ and NbMAPKKK $\gamma$ in cell death induction or plays an essential role in codependent activation of both NbMAPKKK $\beta$ and NbMAPKКK $\gamma$. In contrast, the ion leakage levels induced by transient expression of full-length NbMAPKKK $\alpha$ or NbMAPKKKY were similar in NbMAPKKK $\beta$-silenced plants and control plants (Figure $4 \mathrm{~B}$ ), suggesting that NbMAPKKK $\alpha$ and NbMAPKKK $\beta$ are not codependent in their activation; rather, NbMAPKKK $\beta$ functions upstream of NbMAPKKK $\alpha$ in the cell death signaling pathway. This result led us to postulate that NbMAPKKK $\beta$ functions upstream of NbMAPKKK $\gamma$. As expected, in NbMAPKKK $\gamma$ silenced plants, cell death and the ion leakage level induced by the transient overexpression of the NbMAPKKK $\beta$ kinase domain were significantly compromised compared to control plants (data not shown and Figure $4 \mathrm{C}$, respectively). Cell death and the ion leakage

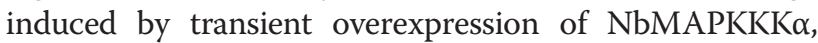
however, were similar in $N b M A P K K K \gamma$-silenced and control plants (data not shown and Figure $4 \mathrm{C}$, respectively), confirming that NbMAPKKK $\beta$ functions upstream of NbMAPKKK $\gamma$ and that NbMAPKKK $\gamma$ functions upstream of $\mathrm{NbMAPKKK} \alpha$. In this epistasis analysis, ion leakage levels were closely associated with the intensity of cell death at all combinations of these genes. Thus, these three MAPKKK genes form a linear signaling pathway leading to PCD in which NbMAPKKK $\beta$ and NbMAPKKK $\alpha$ function as the furthest upstream and downstream components, respectively, of the three MAPKKK components.

\section{Discussion}

In our previous study [19], we showed that a MAPK cascade including NbMAPKKK $\alpha$ and NbMEK2 is required for both the PCD-associated systemic necrosis induced by PlAMV-Li1 and the $R x$-mediated HR against potato virus $\mathrm{X}$. Several studies have demonstrated that at least two MAPK cascades are involved in plant responses to various environmental stimuli $[8,12]$. The great diversity of plant MAPKKK genes is assumed to underlie the ability of plants to mount specific signaling responses to various environmental stimuli $[15,16]$. Therefore, to better understand the functions of MAPKKKs in the induction of PCD, we performed a comparative functional analysis of $N$. benthamiana MAPKKK genes. In the previous and present study, we demonstrated that three of

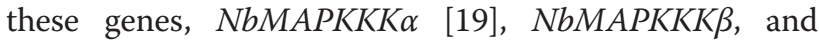
$N b M A P K K K \gamma$ (and/or its homologs), function as positive regulators of PlAMV-Li1-induced PCD and are not functionally redundant in the cell death signaling pathway. In the VIGS experiments, not only $N b M A P K K K \gamma$ but also its homologous gene(s) were presumably knocked down in TRV-NbMAPKKK $\gamma$-infected plants. However, only one gene in the A2 subgroup was obtained from the cloning experiments, suggesting that NbMAPKKK $\gamma$ homologous gene(s), which carries almost the same sequence as $N b M A P K K K \gamma$ itself, must be effectively knocked down in TRV-NbMAPKKK $\gamma$-infected plants. In addition, overexpression of the $N b M A P K K K \gamma$ full-length ORF and kinase domain could induce rapid cell death. Therefore, NbMAPKKK $\gamma$ itself could play an important role in the cell death signaling pathway.

We also explored the functional relationship among these three MAPKKK genes by performing an epistasis experiment based on the assumption that if signaling protein A functions upstream of signaling protein B, signaling by activated $A$ will be suppressed by silencing of B expression, whereas signaling by activated B will not be suppressed by silencing of A expression. Our results (Figure 4) demonstrate that NbMAPKKK $\alpha, \mathrm{NbMAPKKK} \beta$, and NbMAPKKK $\gamma$, which represents NbMAPKKK $\gamma$ and/or its homologous gene(s), form a linear signaling pathway leading to cell death induction in which NbMAPKKK $\beta$ acts upstream of NbMAPKKK $\gamma$ and NbMAPKKK $\gamma$ acts upstream of NbMAPKKK $\alpha$. Results of a similar epistasis analysis have suggested that an NPK1/MEK1/NTF6 MAPK cascade functions downstream of a MAPKKK $\alpha / \mathrm{MEK} 2 /$ SIPK MAPK cascade in the induction of PCD in plants [8]. These results support the possibility that NbMAPKKK $\alpha$, NbMAPKКK $\beta$, NbMAPKKK $\gamma$, and NPK1 initiate four distinct MAPK cascades that are coordinately involved in plant cell death. Alternatively, different sets of MAPK cascades might be involved in cell death signaling pathways induced by different plant-microbe interactions. To investigate this issue, further research is needed to determine whether NbMAPKKK $\beta$ and NbMAPKKK $\gamma$ participate in the $N$ gene-mediated HR against TMV or in the Ptomediated HR against Pst.

In our experiments, the transient overexpression of the NbMAPKKKє2 kinase domain by agroinfiltration did not induce cell death (Figure 2A). However, MelechBonfil and associates have shown that the tomato homolog of NbMAPKKK $€ 2$, SIMAPKKK $\epsilon$, is required for PCD induction in the $\mathrm{HR}$ against Gram-negative bacterial pathogens [9]. Furthermore, they showed that the 

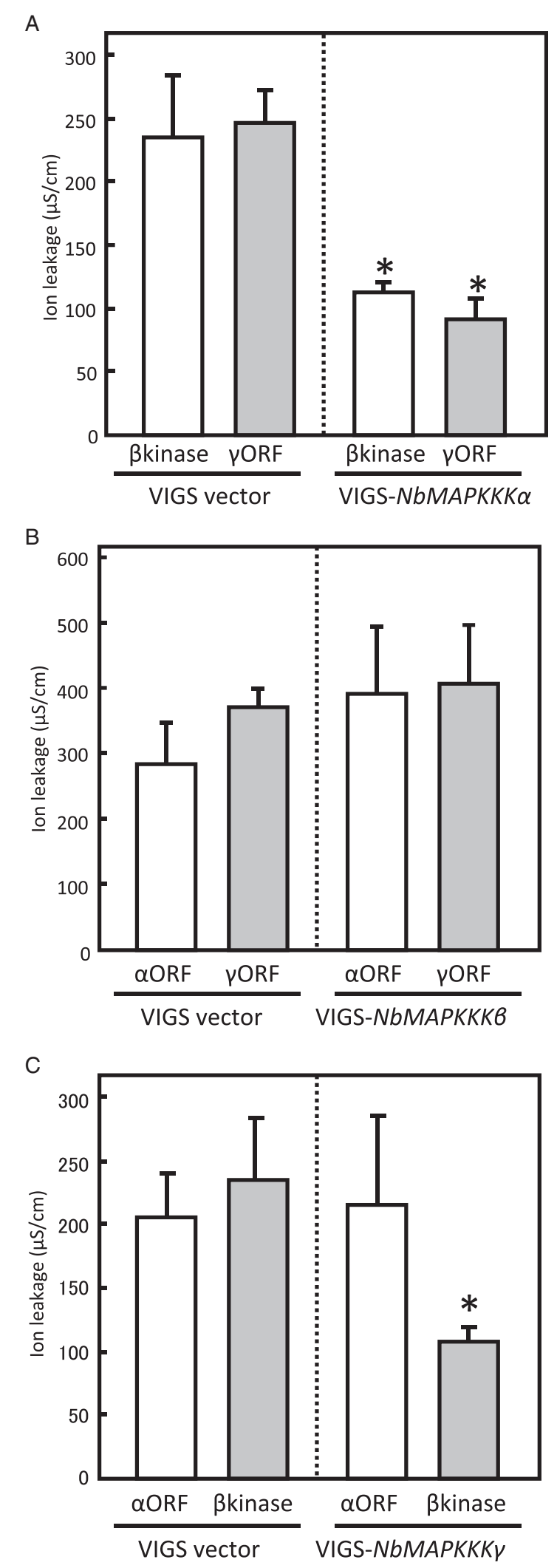

Figure 4 Epistasis analysis of the functional relationships among NbMAPKKKa, NbMAPKKK $\beta$, and NbMAPKKK $\gamma$. Epistasis analysis was performed by combining functional activation and suppression of specific combinations of MAPKKKs using transient overexpression and VIGS, respectively. Cell death in infiltrated areas was quantified using ion leakage assays at $4 \mathrm{dpi}$. Data shown represent means \pm standard deviation for at least five plants. Asterisks indicate statistically significant differences from control plants $(P<0.05)$. All experiments described in this figure were repeated two times with similar results. A) Cell death in the NbMAPKKKß kinase domain (ßkinase)- or NbMAPKKKY (үORF)overexpressing areas of non-silenced and NbMAPKKKa-silenced plants. Agrobacterium cultures expressing $\beta$ kinase and $\mathrm{ORF}$ were grown to a turbidity of 1.0 and 0.05 , respectively, for infiltration. B) Cell death in the NbMAPKKKa (aORF)- or YORF-overexpressing areas of non-silenced and NbMAPKKKB-silenced plants. Agrobacterium cultures expressing aORF and $\mathrm{OORF}$ were grown to a turbidity of 0.5 or 0.05 , respectively, for infiltration. C) Cell death in the aORF- or ßkinase-overexpressing areas of non-silenced and NbMAPKKKYsilenced plants. Agrobacterium cultures expressing aORF and $\beta$ kinase were grown to a turbidity of 0.5 or 1.0 , respectively, for infiltration.

transient overexpression of the tomato SIMAPKKK€ kinase domain in $N$. benthamiana induces pathogenindependent cell death. A comparison between the amino acid sequences of the kinase domains of NbMAPKKK $€ 2$ and SIMAPKKKE indicated only three amino acid differences. These three residues are not highly conserved and are not predicted to be essential for plant kinase catalytic activity, so explaining this functional difference solely by the amino acid sequence level is difficult. Although at first glance, our results seem to contradict theirs, the results cannot be directly compared because three factors in our transient overexpression experiments differed from theirs: promoters, Agrobacterium strains, and species origin of the homologous genes. These three factors, which could influence the expression levels of overexpressed proteins, are critical for the execution of cell death elicited by proteins possessing cell death-inducing activity $[27,28]$. Moreover, the amount of overexpressed NbMAPKKKє2 kinase domain in our experiment was much more than the amounts of NbMAPKKK $\beta$ kinase domain and its $\mathrm{K} \rightarrow \mathrm{M}$ mutant or NbMAPKKK $\gamma$ kinase domain that can induce cell death and was comparable to the amount of the NbMAPKKK $\gamma$ kinase domain $\mathrm{K} \rightarrow \mathrm{M}$ mutant. Therefore, NbMAPKKK $€ 2$ might be a less potent inducer of cell death than other MAPKKKs.

Our suggestion that several MAPK cascades function in a linear signaling pathway in the induction of cell death raises a concern about their roles in plant defense responses. To prevent pathogen invasion, plants commonly employ two types of receptors [NBS-LRR (nucleotide-binding site and leucine-rich repeat) receptors and RLK (receptor-like kinase)-type receptors] [1] to sense multiple PAMPs or effectors derived from biotrophic and necrotrophic pathogens. When plants 
perceive pathogens, the transcription of a common set of genes that act against various types of pathogens is activated $[29,30]$, subsequently producing many defense responses, including cell death and the production of low-molecular-weight signaling compounds such as ethylene and reactive oxygen species (ROS). Activation of the MAPKKK $\alpha / \mathrm{MEK} 2 / \mathrm{SIPK}$ cascade can induce production of ethylene and ROS [31,32]. NPK1, which initiates another MAPK cascade, is activated by the ROS produced by the MAPK cascade initiated by MAPKKK $\alpha$ [26]. Similarly, the alfalfa MAPKKK MsOMTK1 is activated by hydrogen peroxide [6]. These results suggest that compounds induced by an upstream-acting MAPK cascade function as signaling molecules that activate a downstream-acting MAPK cascade. In addition, they suggest that the involvement of several MAPK cascades in defense responses enables plants to activate various responses simultaneously or coordinately to combat various types of pathogens.

\section{Conclusions}

In this study, we demonstrated that three MAPKKKs in $N$. benthamiana form a linear signaling pathway leading to $\mathrm{PCD}$, implying that the involvement of multiple MAPK cascades in plant defense responses enables plants to exhibit various reactions simultaneously. Further analysis to identify downstream MAPKK and MAPK genes directly phosphorylated by NbMAPKKK $\beta$ and NbMAPKKKY should help clarify the regulatory mechanisms of cell death involving these MAPKKK genes. Furthermore, to elucidate the mechanisms underlying the sequential activation of each MAPK cascade during plant defense responses, the upstream components or chemical compounds that directly activate these MAPKKKs must be identified.

\section{Methods}

\section{Plant materials and virus isolate}

$N$. benthamiana plants were grown in a growth chamber at $25{ }^{\circ} \mathrm{C}$. To virally induce $\mathrm{PCD}$, plants were inoculated with the binary vector pLi1, which contains the fulllength cDNA of the Li1 isolate of plantago asiatica mosaic virus (PIAMV) downstream of the cauliflower mosaic virus $35 \mathrm{~S}$ promoter [33].

\section{Cloning of MAPKKK genes from $N$. benthamiana}

To retrieve partial MAPKKK cDNA sequences, a BLAST search was performed using the amino acid sequences of the kinase domains of the Arabidopsis MAPKKK genes AtMEKK1, AtMAPKKK $\gamma$, and AtMAPKKKE1 as queries against the $N$. benthamiana, N. tabacum, and S. lycopersicum databases of the Gene Index Project at the Computational Biology and Functional Genomics Laboratory Web site (http://compbio.dfci.harvard.edu/tgi/cgi-bin/tgi/Blast/ index.cgi) and the tobacco BY-2 EST clone database of the RIKEN BioResource Center (http://www.brc.riken.go.jp/ lab/epd/blast/index.shtml). The consensus nucleotide sequences for the retrieved partial cDNA sequences and query sequences were used to design a pair of specific primers for each of the three MAPKKK genes. Each pair of specific primers was used for RT-PCR amplification of a cDNA fragment from total RNA extracted from $N$. benthamiana leaves. The RT-PCR products were gelpurified and cloned into the pGEM-T easy vector (Promega, Madison, WI, USA) for sequencing. New primers were then designed based on the regions of the obtained cDNA sequences that were nonhomologous to the query sequences (to prevent nonspecific amplification) and used for specific $5^{\prime}$ - and $3^{\prime}$-rapid amplification of cDNA ends (RACE) using a GeneRacer Kit (Invitrogen, Carlsbad, CA, USA) according to the standard protocol provided by the manufacturer. The sequences of primers used in this study are listed in Table 1. cDNA sequences of these MAPKKK genes were determined from at least three independent clones.

ORF and motif analyses were performed using the ORF Finder (http://www.ncbi.nlm.nih.gov/gorf/gorf.html) and CD-Search (http://www.ncbi.nlm.nih.gov/Structure/cdd/ wrpsb.cgi) programs, respectively. Phylogenetic analysis was performed using MEGA3.1 based on a multiple alignment created using ClustalW. The MAPKKK gene sequences used in phylogenetic analysis are listed in the GenBank database under the following accession numbers: AtMEKK1 (NM_116919), AtMEKK2 (NM_116917), AtMEKK3 (NM_116916), AtMEKK4 (NM_117272), BnMAPKKKß1 (AJ010093), AtANP1 (NM_100771), AtANP2 (NM_104370), AtANP3 (NM_111477), NtNPK1 (D26601), AtMAPKKKY (NM_126084), AtYODA

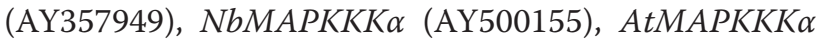
(NM_179472), AtMAPKKKe1 (NM_112199), AtMAPKKKe2 (NM_111677), MsOMTK1 (AJ575100), BnMAPKKKa1

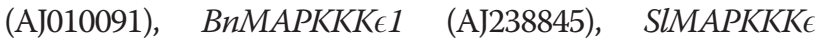
(GU192457), and NbMAPKKKe (GU205153). Homo sapiens HsMEKK1 (AF042838) was used as an outgroup.

Construction of plasmids for transient expression of wildtype and mutant MAPKKKs and MAPKKK kinase domains and agroinfiltration

The coding regions of NbMAPKKK $\alpha, N b M A P K K K \beta$, and $N b M A P K K K \gamma$ were amplified by RT-PCR using primer pairs Kp-NbMAPKKKa-1F/Xh-NbMAPKKKa1836R, Kp-NbMAPKKKb-1F/EcV-NbMAPKKKb-1695R, and Kp-NbMAPKKKg-1F/Xh-NbMAPKKKg-1956R, respectively. The kinase domains of $N b M A P K K K \beta, N b$ $M A P K K K \gamma$, and NbMAPKKK $\epsilon 2$ were amplified using primer pairs Kp-NbMAPKKKbkinase-F/EcV-NbMAPKK Kbkinase-R, Kp-NbMAPKKKgkinase-F/Xh-NbMAPKKK 
Table 1 Primers used in this study

\begin{tabular}{|c|c|c|}
\hline Name & Sequence $\left(5^{\prime}-3^{\prime}\right)$ & Comments \\
\hline NbTC9992-1F & GCTGTCAAAGAAGTGTCATTA & Specific primer for TC9992 \\
\hline NbTC9992-1280R & ACCGTTTATTAATCACTATATTGC & Specific primer for TC9992 \\
\hline NtBP1333-1 F & CTTAATGGGCAAGCAGCTAATC & Specific primer for BP133312 \\
\hline NtBP1333-447R & TCAAGATTGTATGTTGTCTGCTC & Specific primer for BP133312 \\
\hline LeBI9315-123F & GTTGCAATTAAACAAGTTCTCTGGA & Specific primer for B|931567 \\
\hline LeBI9315-658R & GGCTGAAGATCATAGTACGG & Specific primer for B1931567 \\
\hline NbTC9992-458R-5RACE & GCTTGTCCATAGCCTTGGTTCTTCCT & 5'-RACE for TC9992 \\
\hline NbTC9992-49R-5RACE & TITGCCTTCCCCCATCGCCTTGAT & 5'-RACE for TC9992 \\
\hline NbTC9992-F1 & GGATTGGAAAGGGGGAACCT & Sequencing \\
\hline NtMAP3Kb-566F & TCCGCCGGTCATGTCACT & Sequencing \\
\hline NtBP1333-199R-5RACE & ACATGGCTGCAGCTGCTTCATATTC & 5'-RACE for BP133312 \\
\hline NtBP1333-91R-nested & TACTATCCTTCTGCATAACTGACTGCAA & 5'-RACE for BP133312 \\
\hline NtBP1333-333F-5RACE & GAGAATCTCTCATCAGCCAGATGTTC & 3'-RACE for BP133312 \\
\hline NtBP1333-375F-nested & CAAACCTGTTGGTGGGGTACGAAT & 3'-RACE for BP133312 \\
\hline NtBP1333-F1 & AGACGCGCATAATTCGCATC & Sequencing \\
\hline NtBP1333-R1 & TGAGCTCTCGTTTGGTAATAAG & Sequencing \\
\hline LeB19315-507R-5RACE & CATCTGCCTCTGTCAACTTTGTTGCA & 5'-RACE for LeBI931567 \\
\hline LeBI9315-165R-nested & CCTCCTGAGCAATATTCTCCAGAGA & 5'-RACE for LeB1931567 \\
\hline LeB19315-482F-3RACE & TGCAACAAAGTTGACAGAGGCAGATG & 3'-RACE for LeBI931567 \\
\hline LeBI9315-564F-nested & ATGTCGGGAGTATGTGCTGCATCTG & 3'-RACE for LeBI931567 \\
\hline LeBI9315-F1 & CTGAGAAAGTTCTTGCAAACG & Sequencing \\
\hline LeBI9315-F2 & GAACAGATGAATCAGAAGATG & Sequencing \\
\hline LeBI9315-F3 & AAAGCACTCCATATAAACACAG & Sequencing \\
\hline LeBI9315-R1 & GGTATACATCAAGTCCACCAT & Sequencing \\
\hline LeB19315-R2 & GAGGGAGTATGCTCTCATG & Sequencing \\
\hline Kp-NbMAPKKKa-1F & GGGGTACCGAATGCCTGCTTGGTGGGGAA & Full-length ORF of NbMAPKKKa \\
\hline Xh-NbMAPKKKa-1836R & GGCTCGAGTGCTAAAGAATTGGTCTTAGTTITG & Full-length ORF of NbMAPKKKa \\
\hline Kp-NbMAPKKKb-1F & CCGGTACCGAATGCATCGATTGCCAGGAATIITGC & Full-length ORF of NbMAPKKK $\beta$ \\
\hline EcV-NbMAPKKKb-1695R & GGGATATCTTTAAAGCCTCTTGCCCAGATTITG & Full-length ORF of NbMAPKKK $\beta$ \\
\hline Kp-NbMAPKKKg-1F & GGGGTACCGAATGCGTTGGTGGCAGAACG & Full-length ORF of NbMAPKKKY \\
\hline Xh-NbMAPKKKg-1956R & GGCTCGAGTGCTACCTCTCTAGAGATAAACG & Full-length ORF of NbMAPKKKY \\
\hline Kp-NbMAPKKKbkinase-F & CCGGTACCGAATGTACTGGGACAAAGGTGATCT & Kinase domain of NbMAPKKK $\beta$ \\
\hline EcV-NbMAPKKKbkinase-R & GGGATATCTITACACAAAAGGATGCTCCAAGA & Kinase domain of NbMAPKKK $\beta$ \\
\hline Kp-NbMAPKKKgkinase-F & GGGGTACCGAATGTGGCAAAAAGGGAAGCTTATTG & Kinase domain of NbMAPKKKY \\
\hline Xh-NbMAPKKKgkinase-R & GGCTCGAGTGTTACATAAATCGATGTTCCAATAAC & Kinase domain of NbMAPKKKY \\
\hline Kp-NbMAPKKKekinase-F & GGGGTACCGAATGAAATATATGCTCGGAGATGAG & Kinase domain of NbMAPKKKE2 \\
\hline Xh-NbMAPKKKekinase-R & GGCTCGAGTGTTATATCCATGGATGTGAAAGTAG & Kinase domain of NbMAPKKKE2 \\
\hline NbMAPKKKb-K381M-F & TITGCTGTCATGGAAGTGTCATTACTTGATCA & $\mathrm{K} \rightarrow \mathrm{M}$ mutant of NbMAPKKK $\beta$ \\
\hline NbMAPKKKb-K381M-R & ATGACACTTCCATGACAGCAAAAAAGAAACCG & $\mathrm{K} \rightarrow \mathrm{M}$ mutant of NbMAPKKK $\beta$ \\
\hline NbMAPKKKg-K374M-F & CTGGAGCTITATGTGCGATGATGGAAGTTGAATT ATTACCGGA & $\mathrm{K} \rightarrow \mathrm{M}$ mutant of NbMAPKKKY \\
\hline NbMAPKKKg-K374M-R & TCCGGTAATAATTCAACTTCCATCATCGCACATAA AGCTCCAG & $\mathrm{K} \rightarrow \mathrm{M}$ mutant of NbMAPKKKY \\
\hline NbMAPKKKa-255F & GGTTGTITTGGGATGTGGGGTCAG & Real-time RT-PCR for NbMAPKKKa \\
\hline NbMAPKKKa-393R & CAGTGGGCTCAACCTATTATCGCC & Real-time RT-PCR for NbMAPKKKa \\
\hline NbMAPKKKb-1179F & CACAAGGCAGATTITACATGGTTTG & Real-time RT-PCR for NbMAPKKK $\beta$ \\
\hline
\end{tabular}


Table 1 Primers used in this study (Continued)

\begin{tabular}{lll}
\hline NbMAPKKKb-1286R & AGCTTGACCGATCCGTTAGCA & Real-time RT-PCR for NbMAPKKKB \\
NbMAPKKKg-903F & CCGTGAGTGTAGTGCTCAGGGTAA & Real-time RT-PCR for NbMAPKKKY \\
NbMAPKKKg-984R & TGCCGTAGGCTGCTGTGATG & Real-time RT-PCR for NbMAPKKKY \\
Nb18S-193F & ATACGTGCAACAAACCCCGAC & Real-time RT-PCR for Nb18S rRNA \\
Nb18S-280R & TGAATCATCGCAGCAACGG & Real-time RT-PCR for Nb18S rRNA \\
\hline
\end{tabular}

gkinase-R, and Kp-NbMAPKKKekinase-F/Xh-NbMAPK KKekinase-R, respectively.

Loss-of-function mutants of NbMAPKKK $\beta$ and NbMAPKKK $\gamma$ were obtained by substituting methionine for the essential lysine in the ATP-binding site in the kinase domain. These mutants were obtained by sitedirected mutagenesis using primers NbMAPKKKbK381M-F and NbMAPKKKb-K381M-R for NbMAPKKK $\beta$ and NbMAPKKKg-K374M-F and NbMAPKKKg-K374M$\mathrm{R}$ for NbMAPKKK $\gamma$. Each PCR-amplified MAPKKK gene fragment was subcloned into the pEarleyGate 203 vector under $35 \mathrm{~S}$ promoter [34] via the pENTA entry vector [35] with LR Clonase II Enzyme Mix (Invitrogen). GUS gene was also subcloned into the pEarleyGate 203 vector under the $35 \mathrm{~S}$ promoter. These plasmid vectors were transformed into Agrobacterium tumefaciens strain EHA105. Agroinfiltration was performed as previously described [27].

\section{Construction of VIGS vectors and VIGS}

VIGS was performed as described previously using pTV:00 derivatives and pBintra6 [17]. NbMAPKKK $\alpha$ silencing was induced using pTV:NbMAPKKK $\alpha$ [19]. For NbMAPKKK $\beta$ and NbMAPKKKY silencing, the 390-bp KpnI-PvuII fragment of the 5 '-terminal region of the PCR-amplified full-length NbMAPKKK $\beta$ ORF and the 576-bp KpnI-EcoRV fragment of the PCR-amplified full-length NbMAPKKKY ORF were introduced into pTV:00 in the antisense direction to generate pTV: NbMAPKKK $\beta$ and pTV:NbMAPKKK $\gamma$, respectively. The NbMAPKKK $\beta$ ORF was amplified using primers KpNbMAPKKKb-1 F and EcV-NbMAPKKKb-1695R, and the NbMAPKKKY ORF was amplified using primers KpNbMAPKKKg-1 F and Xh-NbMAPKKKg-1956R.

\section{Real-time RT-PCR}

The methods used for total RNA isolation, cDNA synthesis, and quantitative analysis of gene expression were the same as those previously described [18]. Expression of $18 \mathrm{~S}$ rRNA was used as the normalizer. The primers used for realtime RT-PCR were NbMAPKKKa-255F and NbMAPKK Ka-393R for NbMAPKKK $\alpha$, NbMAPKKKb-1179F and NbMAPKKKb-1286R for NbMAPKKK $\beta$, NbMAPKKKg903F and NbMAPKKKg-984R for NbMAPKKK $\gamma$, and Nb18S-193F and Nb280R for Nb18S rRNA.

\section{Additional files}

Additional file 1: Figure S1. Southern blot analysis of A) NbMAPKKK $\beta$ and $\mathbf{B})$ NbMAPKKKY using kinase domain-specific DNA probes. DNA probes were generated by using the PCR DIG Probe Synthesis Kit (Roche, Basel, Switzerland) according to the manufacturer's instructions. Each lane was loaded with $5 \mu \mathrm{g}$ of total genomic DNA digested with each restriction enzyme.

Additional file 2: Figure S2. Ion leakage of GUS-infiltrated areas. An Agrobacterium strain expressing the GUS gene using the $35 \mathrm{~S}$ promoter was infiltrated at the following turbidities: 0.05, 0.5, and 1.0.

\section{Abbreviations}

HR: Hypersensitive response; MAPK: Mitogen-activated protein kinase; MAPKK: MAPK kinase; MAPKKK: MAPKK kinase; PCD: Programmed cell death; PIAMV: plantago asiatica mosaic virus; Pst: Pseudomonas syringae pv. tomato TMV: tobacco mosaic virus.

\section{Authors' contributions}

$\mathrm{MH}, \mathrm{KK}, \mathrm{YY}$ and $\mathrm{SN}$ designed the experiments. $\mathrm{MH}$ and $\mathrm{KK}$ performed the experiments and analyzed the data together with KM, YO, YY and SN. TS, KI and $\mathrm{YT}$ contributed new reagents and analytic tools. $\mathrm{MH}, \mathrm{KK}$ and $\mathrm{SN}$ wrote the paper. All authors discussed the results and approved the final manuscript.

\section{Acknowledgements}

We thank Dr. David Baulcombe (University of Cambridge, Cambridge, UK) for tobacco rattle virus vector and silencing suppressor p19 gene. This work supported by grand-in-aid from the Japan Society for the Promotion of Science and, in part, by a Grand-in-aid for JSPS Fellows, and by the Program for Promotion of Basic Research Activities for Innovative Bioscience (PROBRAIN).

Received: 19 March 2012 Accepted: 26 June 2012

Published: 8 July 2012

\section{References}

1. Jones JD, Dangl JL: The plant immune system. Nature 2006, 444 (7117):323-329.

2. Rogers HJ: Cell death and organ development in plants. Curr Top Dev Biol 2005, 71:225-261.

3. Rodriguez MC, Petersen M, Mundy J: Mitogen-activated protein kinase signaling in plants. Annu Rev Plant Biol, 61:621-649.

4. MAPKGroup: Mitogen-activated protein kinase cascades in plants: a new nomenclature. Trends Plant Sci 2002, 7(7):301-308.

5. Asai T, Tena G, Plotnikova J, Willmann MR, Chiu WL, Gomez-Gomez L, Boller T, Ausubel FM, Sheen J: MAP kinase signalling cascade in Arabidopsis innate immunity. Nature 2002, 415(6875):977-983.

6. Nakagami H, Kiegerl S, Hirt H: OMTK1, a novel MAPKKK, channels oxidative stress signaling through direct MAPK interaction. J Biol Chem 2004, 279(26):26959-26966.

7. Jin H, Axtell MJ, Dahlbeck D, Ekwenna O, Zhang S, Staskawicz B, Baker B: NPK1, an MEKK1-like mitogen-activated protein kinase kinase kinase, regulates innate immunity and development in plants. Dev Cell 2002, 3 (2):291-297.

8. del Pozo O, Pedley KF, Martin GB: MAPKKKa is a positive regulator of cell death associated with both plant immunity and disease. EMBO J 2004, 23 (15):3072-3082. 
9. Melech-Bonfil S, Sessa G: Tomato MAPKKKє is a positive regulator of celldeath signaling networks associated with plant immunity. Plant J 2010, 64(3):379-391

10. Jin H, Liu Y, Yang KY, Kim CY, Baker B, Zhang S: Function of a mitogenactivated protein kinase pathway in $\mathrm{N}$ gene-mediated resistance in tobacco. Plant J 2003, 33(4):719-731.

11. Ekengren SK, Liu Y, Schiff M, Dinesh-Kumar SP, Martin GB: Two MAPK cascades, NPR1, and TGA transcription factors play a role in Ptomediated disease resistance in tomato. Plant J 2003, 36(6):905-917.

12. Yoo SD, Cho YH, Tena G, Xiong Y, Sheen J: Dual control of nuclear EIN3 by bifurcate MAPK cascades in C2H4 signalling. Nature 2008, 451(7180):789-795.

13. Liu Y, Zhang S: Phosphorylation of 1-aminocyclopropane-1-carboxylic acid synthase by MPK6, a stress-responsive mitogen-activated protein kinase, induces ethylene biosynthesis in Arabidopsis. Plant Cell 2004, 16(12):3386-3399.

14. Wang H, Ngwenyama N, Liu Y, Walker JC, Zhang S: Stomatal development and patterning are regulated by environmentally responsive mitogenactivated protein kinases in Arabidopsis. Plant Cell 2007, 19(1):63-73.

15. Zhang S, Klessig DF: MAPK cascades in plant defense signaling. Trends Plant Sci 2001, 6(11):520-527.

16. Pedley KF, Martin GB: Role of mitogen-activated protein kinases in plant immunity. Curr Opin Plant Biol 2005, 8(5):541-547.

17. Ratcliff F, Martin-Hernandez AM, Baulcombe DC: Tobacco rattle virus as a vector for analysis of gene function by silencing. Plant J 2001, 25(2):237-245.

18. Komatsu K, Yamaji Y, Ozeki J, Hashimoto M, Kagiwada S, Takahashi S, Namba S: Nucleotide sequence analysis of seven Japanese isolates of Plantago asiatica mosaic virus (PIAMV): a unique potexvirus with significantly high genomic and biological variability within the species. Arch Virol 2008, 153(1):193-198.

19. Komatsu K, Hashimoto M, Ozeki J, Yamaji Y, Maejima K, Senshu H, Himeno M, Okano Y, Kagiwada S, Namba S: Viral-induced systemic necrosis in plants involves both programmed cell death and the inhibition of viral multiplication, which are regulated by independent pathways. Mol Plant Microbe Interact 2010, 23(3):283-293.

20. Mizoguchi T, Irie K, Hirayama T, Hayashida N, Yamaguchi-Shinozaki K, Matsumoto K, Shinozaki K: A gene encoding a mitogen-activated protein kinase kinase kinase is induced simultaneously with genes for a mitogen-activated protein kinase and an $\mathbf{S 6}$ ribosomal protein kinase by touch, cold, and water stress in Arabidopsis thaliana. Proc Natl Acad Sci USA 1996, 93(2):765-769.

21. Jouannic S, Champion A, Segui-Simarro JM, Salimova E, Picaud A, Tregear J, Testillano P, Risueno MC, Simanis $V$, Kreis $M$, et al: The protein kinases

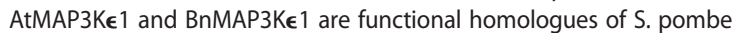
cdc7p and may be involved in cell division. Plant J 2001, 26(6):637-649.

22. Chaiwongsar S, Otegui MS, Jester PJ, Monson SS, Krysan PJ: The protein kinase genes MAP3Kє1 and MAP3Kє2 are required for pollen viability in Arabidopsis thaliana. Plant J 2006, 48(2):193-205.

23. Banno H, Hirano K, Nakamura T, Irie K, Nomoto S, Matsumoto K, Machida Y: NPK1, a tobacco gene that encodes a protein with a domain homologous to yeast BCK1, STE11, and Byr2 protein kinases. Mol Cell Biol 1993, 13(8):4745-4752.

24. Ren D, Yang KY, Li GJ, Liu Y, Zhang S: Activation of Ntf4, a tobacco mitogen-activated protein kinase, during plant defense response and its involvement in hypersensitive response-like cell death. Plant Physiol 2006 141(4):1482-1493.

25. Zhang S, Liu Y: Activation of salicylic acid-induced protein kinase, a mitogen-activated protein kinase, induces multiple defense responses in tobacco. Plant Cell 2001, 13(8):1877-1889.

26. Kovtun Y, Chiu WL, Tena G, Sheen J: Functional analysis of oxidative stress-activated mitogen-activated protein kinase cascade in plants. Proc Natl Acad Sci USA 2000, 97(6):2940-2945

27. Komatsu K, Hashimoto M, Maejima K, Shiraishi T, Neriya Y, Miura C, Minato N, Okano Y, Sugawara K, Yamaji Y, Namba S: A necrosis-inducing elicitor domain encoded by both symptomatic and asymptomatic Plantago asiatica mosaic virus isolates, whose expression is modulated by virus replication. Mol Plant Microbe Interact 2011, 24(4):408-420.

28. Zhang Y, Dorey S, Swiderski M, Jones JD: Expression of RPS4 in tobacco induces an AvrRps4-independent HR that requires EDS1, SGT1 and HSP90. Plant J 2004, 40(2):213-224.

29. Navarro L, Zipfel C, Rowland O, Keller I, Robatzek S, Boller T, Jones JD: The transcriptional innate immune response to flg22. Interplay and overlap with Avr gene-dependent defense responses and bacterial pathogenesis. Plant Physiol 2004, 135(2):1113-1128.

30. Tao Y, Xie Z, Chen W, Glazebrook J, Chang HS, Han B, Zhu T, Zou G, Katagiri F: Quantitative nature of Arabidopsis responses during compatible and incompatible interactions with the bacterial pathogen Pseudomonas syringae. Plant Cell 2003, 15(2):317-330.

31. Kim CY, Liu Y, Thorne ET, Yang H, Fukushige H, Gassmann W, Hildebrand D, Sharp RE, Zhang S: Activation of a stress-responsive mitogen-activated protein kinase cascade induces the biosynthesis of ethylene in plants. Plant Cell 2003, 15(11):2707-2718.

32. Liu Y, Ren D, Pike S, Pallardy S, Gassmann W, Zhang S: Chloroplastgenerated reactive oxygen species are involved in hypersensitive response-like cell death mediated by a mitogen-activated protein kinase cascade. Plant J 2007, 51(6):941-954.

33. Ozeki J, Takahashi S, Komatsu K, Kagiwada S, Yamashita K, Mori T, Hirata H, Yamaji Y, Ugaki M, Namba S: A single amino acid in the RNA-dependent RNA polymerase of Plantago asiatica mosaic virus contributes to systemic necrosis. Arch Virol 2006, 151(10):2067-2075.

34. Earley KW, Haag JR, Pontes O, Opper K, Juehne T, Song K, Pikaard CS: Gateway-compatible vectors for plant functional genomics and proteomics. Plant J 2006, 45(4):616-629.

35. Himeno M, Maejima K, Komatsu K, Ozeki J, Hashimoto M, Kagiwada S, Yamaji Y, Namba S: Significantly low level of small RNA accumulation derived from an encapsidated mycovirus with dsRNA genome. Virology, 396(1):69-75.

\section{doi:10.1186/1471-2229-12-103}

Cite this article as: Hashimoto et al:: Identification of three MAPKKKs forming a linear signaling pathway leading to programmed cell death in Nicotiana benthamiana. BMC Plant Biology 2012 12:103.

\section{Submit your next manuscript to BioMed Central and take full advantage of:}

- Convenient online submission

- Thorough peer review

- No space constraints or color figure charges

- Immediate publication on acceptance

- Inclusion in PubMed, CAS, Scopus and Google Scholar

- Research which is freely available for redistribution 